\title{
Quantification of Spatial Structure of Human Proximal Tibial Bone Biopsies Using 3D Measures of Complexity
}

\author{
Peter I. Saparin ${ }^{\mathrm{a}, *}$, Jesper Skovhus Thomsen ${ }^{\mathrm{b}}$, \\ Steffen Prohaska ${ }^{\mathrm{c}}$, Alexei Zaikin ${ }^{\mathrm{d}}$, Jürgen Kurths ${ }^{\mathrm{d}}$, \\ Hans-Christian Hege ${ }^{c}$, Wolfgang Gowin ${ }^{a}$ \\ ${ }^{a}$ Center of Muscle and Bone Research, Dept. of Radiology and Nuclear Medicine, \\ Charité - University Medicine Berlin, Campus Benjamin Franklin, \\ Hindenburgdamm 20, 12203 Berlin, Germany \\ ${ }^{\mathrm{b}}$ Department of Connective Tissue Biology, Institute of Anatomy, University of \\ Aarhus, DK-8000 Arhus C, Denmark \\ ${ }^{\mathrm{c}}$ Zuse Institute Berlin (ZIB), Scientific Visualization Department, Takustr. 7, \\ 14195 Berlin, Germany \\ ${ }^{\mathrm{d}}$ Center for Dynamics of Complex Systems, Institute of Physics, University of \\ Potsdam, Am Neuen Palais 10, 14415, Potsdam, Germany
}

\begin{abstract}
Changes in trabecular bone composition during development of osteoporosis are used as a model for bone loss in microgravity conditions during a space flight. Symbolic dynamics and measures of complexity are proposed and applied to assess quantitatively the structural composition of bone tissue from 3D data sets of human tibia bone biopsies acquired by a micro-CT scanner. In order to justify the newly proposed approach, the measures of complexity of the bone architecture were compared with the results of traditional $2 \mathrm{D}$ bone histomorphometry. The proposed technique is able to quantify the structural loss of the bone tissue and may help to diagnose and to monitor changes in bone structure of patients on Earth as well as space-flying personnel.
\end{abstract}

Key words: 3D image analysis, measures of complexity, micro-CT, osteoporosis, histomorphometry

PACS: 87.57.-s, 87.59.Fm, 81.70.Ex

\footnotetext{
* Corresponding author

Email address: peter.saparin@charite.de (Peter I. Saparin).

$U R L:$ http://bone3D.zib.de (Peter I. Saparin).
} 


\section{Introduction}

Recent studies have confirmed negative changes in bone composition of space flying personnel during their missions [1-3]. The most typical changes in the bone tissue under microgravity conditions include the loss of bone mass, and the reduction of bone strength $[1,4]$. These skeletal alterations can consequently lead to a development of osteopenia and osteoporosis and to an increased risk of bone fracture.

We utilize the architectural changes in trabecular bone structure that occur during development of osteoporosis as a model for bone loss in microgravity conditions during space flight and propose new measures of complexity to quantify these structural changes. The aim of the study was to assess the deterioration of the 3D structural composition of human trabecular bone in osteoporosis using 3D data sets of human tibial bone biopsies acquired by micro-CT. We propose that the structure of the bone can be regarded as a complex system [5-7] and apply measures of complexity to analyze quantitatively the 3D structural composition of bone tissue. Complexity characterizes a system with many interacting and interrelating components. These interactions and transactions lead to the emergence of new collective nonlinear properties of the system as a whole. The collective behavior of certain parts of the system implies that this activity is a property of the whole system, but not a property of its single parts [8,9]. Another definition of complexity states that it is the property of a real world system that manifests the inability of any one formalism alone being adequate to capture all its properties [10]. Thus, a set of different measures of complexity is required to quantify different aspects of complex nonlinear systems.

We applied symbolic dynamics [11-13] and measures of complexity $[13,14]$ to assess quantitatively the structural composition of bone tissue in 3D. Originally, these methods were developed and successfully applied for quantification of 2D bone structure from CTimages [14-16]. Now we have further extended these approaches and developed symbolic encoding procedures and measures of complexity which are able to quantify the 3D structural composition of bone tissue. We used standardized volumes of interest (VOI) in order to compare the structure of different biopsies, performed quantitative assessments, and made comparisons of the bone architecture as well as of the amount of bone material from different tibia biopsies. In order to justify the approach, the measures of complexity of the bone architecture were compared with the results of traditional 2D bone histomorphometry [17-23].

The evaluation of bone biopsies in general is cardinal and valuable for the study of architectural bone changes in clinical studies and for the validation of any new noninvasive quantitative technique. This study is essential, in addition to the introduction of new 3D structural parameters, to establish the proximal tibia as a new site for noninvasive radiological quantitative assessment. 


\section{Materials and Methods}

\subsection{Biopsy acquisition}

The investigation used a bone-healthy (no bone diseases except some subjects having osteoporosis) autopsy material comprising 16 women aged 57-92 years and 8 men aged 60-94 years. 24 bone biopsies were taken $15 \mathrm{~mm}$ below the tibia plateau at the medial side. The biopsies were obtained with a surgical diamond tipped coring drill with utmost care and the best possible precision. The biopsies have a shape of a cylinder with a diameter of $7 \mathrm{~mm}$. The length of the biopsies varies between 20 and $40 \mathrm{~mm}$.

While the iliac crest is the normal site for diagnostic bone biopsies, this skeletal site is not suited for studying the influence of microgravity as it is not weight bearing to the same degree as e.g. the proximal tibial metaphysis or the vertebral body. Even if the proximal tibial metaphysis is not routinely in clinical use for diagnostic bone biopsying it is used as a donor site for trabecular bone grafting [24-26].

\subsection{Scanning with micro-CT}

The biopsies were embedded in methylmetacrylate resulting in $14 \mathrm{~mm}$ wide plastic cylinders. The biopsies were scanned with a Scanco $\mu \mathrm{CT} 40$ micro-CT scanner at Scanco Medical AG, Switzerland, using a voxel size of $20 \times 20 \times 20 \mu \mathrm{m}$.

\subsection{Histomorphometry}

After micro-CT scanning, the biopsies were sectioned, stained, mounted, and evaluated by traditional 2D histomorphometry. Sixteen 10- $\mu$ m-thick sections grouped in 8 disector pairs [17] were cut from the central $2 \mathrm{~mm}$ of the biopsies. The sections were digitized by use of a flatbed image scanner with an integrated transparency scanning unit, using a pixel size of $10 \mu \mathrm{m} \times 10 \mu \mathrm{m}$. Static histomorphometry was performed by custom-made software, which has previously been described in details [18]. The following parameters were measured: $2 \mathrm{D}$ trabecular bone volume $\mathrm{BV} / \mathrm{TV}_{2 \mathrm{D}}$; marrow and bone space star volume $V_{\mathrm{m} . s p a c e}^{*}$ and $V_{\mathrm{b} . \text { space }}^{*}[19]$; trabecular thickness Tb.Th, trabecular number Tb.N, and trabecular separation Tb.Sp [20]; node-terminus ratio Nd/Tm [21]; trabecular bone pattern factor TBPf [22]; and connectivity density CD [23]. For every analyzed section, a $2 \mathrm{D}$ region of interest (ROI) was selected to match its corresponding 3D VOI, which is chosen as described in Section 3.1. 


\subsection{Assessment of 3D structural composition}

Our technique to quantify the structural composition of the biopsies consists of four main stages:

(1) Selection of volume of interest within the biopsy. The aim of this step is to specify a volume which is free from residual drilling dust (Fig. 1), and to select a standardized region within the biopsy.

(2) Assessment of bone tissue from raw micro-CT data (x-ray attenuation) or from the binarized images. At this stage we calculate the bone volume to total volume ratio in $3 \mathrm{D}\left(\mathrm{BV} / \mathrm{TV}_{3 \mathrm{D}}\right)$, which characterizes the amount of material used in spatial bone construction.

(3) Symbol-encoding of 3D data. This preserves the robust and crucial information about the original topological structure but dramatically decreases the amount of information to be processed to quantify its structural organization. Only a few different symbols are used for the encoding.

(4) Assessment of the bone structure from symbol-encoded images is the final stage of our procedure. The structural composition which is now represented by the spatial arrangement of symbols is quantified by a set of measures of complexity.

\subsubsection{D symbol-encoding}

The purpose of the symbol-encoding procedure is to reduce the amount of information in a $3 \mathrm{D}$ micro-CT data set to its essential structural composition by representing the bone architecture using a very limited set of structural elements or symbols. Our initial intention was to use the same symbolic encoding procedure which we applied to analyze 2D CT-images [14-16]. It was based on an alphabet of five different symbols: three static and two dynamic symbols. However, we found that the edge definition and the distribution of attenuation within the trabeculae of the 3D micro-CT data sets was not as sharp and well defined as the one obtained by a high-resolution 2D CT. Under these circumstances the five-symbol-encoding method is too sensitive.

The modified symbol-encoding procedure for the micro-CT data is based on an alphabet of three different symbols: M, marrow voxel; I, internal bone voxel; and S, surface bone voxel. The two first symbols are static, while the last one, $\mathrm{S}$, is transitional (dynamic). Instead of being based on dynamics of x-ray attenuation only, like in the $2 \mathrm{D}$ technique $[14,15]$, the "modified" transitional symbol combines geometrical and attenuation information. Bone "surface" is a one voxel-thick layer of bone voxels which are lying at the boundary between two tissues: bone and marrow. Therefore, the encoding procedure still uses a mixture of static and transitional symbols.

\subsubsection{D Measures of Complexity}

We have developed and tested several different approaches to quantify and visualize the spatial architecture of trabecular bone using micro-CT scans, see [27-29]. Investigation 
of 3D measures of complexity is part of our ongoing research project and must therefore be considered as work in progress. This report presents the most relevant and promising measures:

- 3D Normalized Entropy of geometrical locations of bone tissue, $S_{\text {norm }}$;

- Structure Complexity Index based on 3D distribution of local spatial ratios of bone volume to total volume $\left(\mathrm{BV} / \mathrm{TV}_{\text {loc }}\right) \mathrm{SCI}\left(\mathrm{BV} / \mathrm{TV}_{\text {loc }}\right)$;

- Structure Complexity Index, SCI3D;

- Surface Complexity Index, SurfCI;

- Surface Index of Global Ensemble, SurfIGE.

The 3D Normalized Entropy of geometrical locations and the SCI based on distribution of local BV/TV-indices can be calculated either from raw micro-CT data or from binarized images. The three other measures, SCI3D, SurfCI, and SurfIGE are based on 3D symbolencoded data.

3D Normalized Entropy of geometrical locations of bone tissue $S_{\text {norm }}$ assesses the spatial distribution of bone material in the VOI. It is calculated as follows (see Fig. 2): The 3D VOI is split into a certain number of cubic bins using an equidistant partition. The probability to find a bone voxel in every cell $p(i, j, k)$ is then calculated and the probability density distribution is constructed. This distribution is normalized so that the sum of all probability densities is 1 . This spatial density distribution can be quantified by the value of its 3D entropy $S$, in generalization of the Shannon entropy of an 1D distribution $[30,31]$

$$
S=-\sum_{i, j, k}^{\text {VOI }} p(i, j, k) \cdot \log _{2}[p(i, j, k)], \text { where } \sum_{i, j, k}^{\text {VOI }} p(i, j, k)=1 \text {. }
$$

Here indices $i, j, k$ are integer positions of the bins within the partitions of three orthogonal coordinate axes (Fig. 2, left). The value of the entropy, calculated in such a way, depends on the partition of the VOI (size and number of bins) and on the shape of the VOI that restricts which cells of the partition can be occupied by bone voxels. Thus, the necessity of a normalization of the entropy arises. As a normalization factor, we use the maximal value of entropy possible for the chosen partition of the $3 \mathrm{D}$ space and for the particular shape of the VOI (Fig. 2, right). Both factors define the maximal number of cells able to contain bone voxels. The maximal possible value of entropy is given by

$$
S_{\max }=\log _{2}\left(N_{\text {bins }} \subset \mathrm{VOI}\right)
$$

The normalized entropy is the entropy of geometrical locations of bone (Eq. 1) tissue normalized by the maximal possible entropy for the given partition of 3D space and for the given shape of the VOI (Eq. 2):

$$
S_{\text {norm }}=-\sum_{i, j, k} p(i, j, k) \cdot \log _{2}[p(i, j, k)] / \log _{2}\left(N_{\text {bins }} \subset \mathrm{VOI}\right) .
$$

The calculation of complexity indices SCI3D, SCI(BV/TV loc $)$, and $\mathbf{S u r f C I}$ is based on a cubic window moving through the VOI (Fig. 3). Local statistics are collected in every 
position of the cubic window and the distributions of local quantities $\hat{p}(\ldots)$ are calculated. After the cubic window has moved thru the entire VOI, the distribution of local statistics for 3D VOI is constructed and quantified. Depending on which local statistics $\hat{p}(\ldots)$ are analyzed and what kind of distribution is constructed, it is possible to quantify different aspects of the bone architecture.

The complexity of the distribution of local $\mathrm{BV} / \mathrm{TV}$ ratios within the VOI is qualified by $\mathrm{SCI}\left(\mathrm{BV} / \mathrm{TV}_{\text {loc }}\right)$ :

$$
\mathrm{SCI}\left(\mathrm{BV} / \mathrm{TV}_{\mathrm{loc}}\right)=-\sum^{\mathrm{VOI}} \hat{p}_{\mathrm{BV} / \mathrm{TV}_{\mathrm{loc}}} \cdot \log _{2} \hat{p}_{\mathrm{BV} / \mathrm{TV}_{\mathrm{loc}}}
$$

where $\mathrm{BV} / \mathrm{TV}_{\text {loc }}$ is the bone volume to total volume ratio at the current location of the moving cubic window, and $\hat{p}_{\mathrm{BV} / \mathrm{TV}_{\text {loc }}}$ is the probability density distribution of local $\mathrm{BV} / \mathrm{TV}$ values.

The complexity of a bone structural composition as a whole is assessed by the Spatial Structure Complexity Index SCI3D:

$$
\begin{aligned}
\mathrm{SCI} 3 \mathrm{D} & =-\sum_{\mathrm{VOI}} \hat{p}_{\mathrm{ILE3D}} \cdot \log _{2} \hat{p}_{\mathrm{ILE3D}}, \quad \text { where } \\
\mathrm{ILE} 3 \mathrm{D} & =\left[p_{\mathrm{loc}}(I)+p_{\mathrm{loc}}(S)\right] /\left[p_{\mathrm{loc}}(M)+\varepsilon\right] .
\end{aligned}
$$

Here $\hat{p}_{\text {ILE3D }}$ is the probability density distribution of indices of a local ensemble (ILE) (see $[14]) ; p_{\text {loc }}(I), p_{\text {loc }}(S)$, and $p_{\text {loc }}(M)$ are the probabilities of symbols representing internal bone, bone surface and marrow voxels correspondingly, and $\varepsilon$ is the predefined neglectably small constant to avoid devision by zero. ILE represents the degree of interstratification of different levels of attenuations and is also the ratio between positive and negative structural element of the bone within the current location of the analyzed cubic window.

SurfCI quantifies the complexity of the spatial distribution of bone surface voxels within the analyzed VOI:

$$
\begin{aligned}
\text { SurfCI } & =-\sum \hat{p}_{\text {surf }} \cdot \log _{2} \hat{p}_{\text {surf }}, \quad \text { where } \\
p_{\text {surf }} & =\left[p_{\text {loc }}(S)\right] /\left[p_{\text {loc }}(I)+p_{\text {loc }}(S)\right] .
\end{aligned}
$$

$\hat{p}_{\text {surf }}$ is the distribution of local surface indices which are introduced by Eq. 8. As in Eq. $5,6, p_{\text {loc }}(I), p_{\text {loc }}(S)$, and $p_{\text {loc }}(M)$ are the probabilities of symbols representing internal bone, bone surface, and marrow voxels respectively.

In order to keep the formulae clear, the normalization conditions for the distribution of probability densities and for the entropies are not included in Eqs. 4-8. In addition to the presented formulae, every constructed distribution of the probability density is normalized as it is done in Eq. 2, and every calculated value of entropy is normalized according to Eq. 3. 
The Surface Index of Global Ensemble, (SurfIGE) is introduced similar to the calculation of local surface indices (Eq. 8), but omits the use of the moving cubic window. Instead, it uses the probabilities of different symbols calculated from the entire VOI.

The BV/TV ratio provides an assessment of the amount of bone material used to construct the analyzed spatial trabecular structure. It is calculated in addition to the structural measures (Eqs. 3-8) exactly from the same VOI which is used for the complexity evaluation. In the present paper the $\mathrm{BV} / \mathrm{TV}$ always denotes the $3 \mathrm{D}$ ratio calculated from the biopsy data, if not explicitly specified otherwise.

\section{Results and discussion}

\subsection{Structural differences within the biopsy. Standardization of VOI.}

When Fig. 4 is inspected it is clear that the trabecular bone density (BV/TV) varies with the distance from the cortical shell. The part of the biopsy that is most distant from the cortical shell, i.e. is placed most central in the knee, is substantial less dense than the region closer to the cortex. Due to the technique of acquiring a bone biopsy, a variation in length of the analyzed tibia bone biopsies is unavoidable. Thus, two issues should be considered before one can compare the structure of different biopsies: 1) How big are the differences in structural composition of different regions within the same biopsy? 2) How to perform an optimal and standardized selection of a VOI within the analyzed biopsies?

In order to answer these questions the following experiment was performed. The three longest biopsies were selected, and every biopsy was numerically segmented into fragments of $5 \mathrm{~mm}$ length starting from the cortical bone as illustrated in Fig. 4. Measures of complexity and BV/TV were calculated from every fragment and plotted versus the serial number of the fragment; the results are shown in Fig. 5. We found that the structure of the biopsy changes dramatically within the interval of $20-25 \mathrm{~mm}$ : BV/TV changes $1.5-2$ times and the complexity changes nearly two times. Thus, the structural composition of the bone should not be compared by using entire biopsies of different lengths. It is necessary to choose a standardized location and size of the VOI in every biopsy in order to be able to compare their architecture quantitatively.

We decided to take the region where the BV/TV and complexity measures showed the lowest changes in biopsy structural composition as a standardized VOI. This is a $10 \mathrm{~mm}$ long segment located $5 \mathrm{~mm}$ below the cortical bone (all measurements are made along the main biopsy axis).

To confirm the stability of the results we checked how a small shift in a positioning of this $1 \mathrm{~cm}$ long VOI affect the evaluation results. The VOI was shifted between $-2 \mathrm{~mm}$ and $+2 \mathrm{~mm}$ from its desired location in 5 steps. Measures of complexity and BV/TV were calculated for every location of the VOI. The direction of shift towards the cortical shell results in less than $3 \%$ changes in the structural parameters. The shift in the opposite direction, towards the inner part of the bone resulted in more than $6 \%$ changes in the 
structural measures quantifying the bone composition. Thus, when selecting the VOI and in a case of uncertainty, it is better to err towards the cortical bone in the positioning of the VOI.

Furthermore, a thin region along the edges of the biopsies were excluded from the VOI in order to avoid drilling residue. However, the amount of drilling residue varies between the biopsies and the size of the VOI therefore varies slightly between the biopsies. We found that the size of the VOI varied at maximum $6 \%$ from its average value of $366.4 \mathrm{~mm}^{3}$. So, the proposed procedure to select and standardize the analyzed VOIs works very well. Significant variations in the absolute sizes of the VOIs are not to be expected.

\subsection{Complexity of 3D bone composition versus bone mass.}

Using the developed technique of standardized VOI selection, symbol-encoding, and quantification by measures of complexity, we performed a 3D evaluation of the structural composition of 24 biopsies. To analyze the changes in bone composition during development of osteoporosis, the results of the biopsy evaluation were represented on bone structure complexity versus bone tissue amount (assessed by BV/TV) diagrams.

We found that the spatial complexity of bone architecture decreases with a loss of the bone density (Fig. 6). This is confirmed by two independent measures: by the Normalized Entropy of geometrical locations and by the SCI3D. Shannon entropy of the distributions is the "classic" measure of complexity [30,31]. Normalized Entropy is directly derived from the Shannon entropy and it is used to verify the results provided by newly developed measures such as SCI3D. The comparison of Normalized Entropy $S_{\text {norm }}$ and SCI3D plots clearly shows the advantage of the symbolic encoding and the 3D moving cubic window approach: the range of change of the SCI $(36 \%)$ is more than 5 times larger than the one of the $S_{\text {norm }}(7 \%)$.

The Structure Complexity Index based on the distribution of local BV/TV ratios, ( $\mathrm{SCI}\left(\mathrm{BV} / \mathrm{TV}_{\text {loc }}\right)$, Fig 7$)$, does not utilize the symbol encoding procedure and is some kind of a "bridge" between traditional histomorphometry and the measures of complexity. The $25 \%$ decrease of SCI(BV/TV) confirms that the bones with a lower bone density have a less complex structure and indicates that in these biopsies the local amounts of bone material are more evenly distributed within the biopsies.

The Surface Complexity Index (SurfCI) (Fig. 8, left) assesses the complexity of the distribution of bone surface voxels in 3D. This measure shows that the maximal complexity is achieved in the bones with the highest bone densities. SurfCI also shows that the distribution of surfaces is less locally homogeneous in biopsies with high BV/TV values.

The next measure, Surface Index of Global Ensemble, quantifies the relative probability of surface voxels within the entire 3D VOI (Fig. 8, right). It indicates that the relative amount of surface bone voxels increases when bone mass is lost: SurfCI grows when $\mathrm{BV} / \mathrm{TV}$ decreases. In order to check this finding, we explicitly plotted the relative probability of surface (S) and internal (I) bone voxels versus BV/TV (Fig. 9). "Relative" 
means here that we calculate the probability of surface or internal bone voxel relatively to the total amount of bone voxels. While the absolute probability of both symbols $\mathrm{S}$ and I decrease when bone mass is lost, beginning at $\mathrm{BV} / \mathrm{TV} \approx 17-19 \%$, they decrease at a different rate. This effect is better represented by the relative probabilities, (Fig. 9). The relative probability of surface voxel increases, while the relative probability of internal bone voxel decreases, thus capturing the difference in bone architecture. When bone mass is lost the trabeculae get thinner and a relative large part of bone voxels is then occupied by surface voxels. It is interesting that the point where these probabilities start to behave differently corresponds to the QCT-derived BMD of 80 to $100 \mathrm{mg} / \mathrm{cm}^{3}$ (a BMD-value that is between "normal" and "osteoporotic").

\subsection{Correlation between different measures and approaches}

In the reported study, we used three different approaches to quantify the structure of the proximal tibia metaphysis. We applied measures of complexity derived from the 3D analysis of small bone samples, the biopsies. Next, the biopsies were analyzed using the 2D technique of histomorphometry. Furthermore, the entire proximal tibia specimens were analyzed by acquiring axial peripheral quantitative computed tomography (pQCT) slices through the entire bones. Every approach provided a set of quantitative parameters about the same bone. In order to study the correlation between the different parameters they were compared pairwise by using the Spearman's rank-order correlation coefficient $r_{s}$. The correlation between two measures was considered to be good if $r_{s}>0.6$.

Three different approaches were applied to evaluate the amount of material used to construct the bone: 3D BV/TV assessment provided by micro-CT, 2D BV/TV provided by the biopsy histomorphometry (both are biopsy based), and the bone mineral density (BMD) provided by peripheral quantitative computed tomography (pQCT, see [16] for details). The last technique uses the entire $2 \mathrm{D}$ slices through the same regions of the proximal tibiae which were used to collect biopsies. We found a very good correlation between all measures quantifying the amount of bone material used to construct the bone. The measures obtained from the 3D analysis of the biopsies are perfectly correlated to the assessment based on $2 \mathrm{D}$ histomorphometry $\left(r_{s}=0.96\right)$, as well as to the BMD estimations obtained by analyzing the axial slices thru the entire bone $\left(r_{s}=0.88\right.$ and 0.86 for cortical plus trabecular bone BMD and trabecular bone BMD respectively). Such a high value of correlation indicates that any of the analyzed measures, the $3 \mathrm{D} \mathrm{BV} / \mathrm{TV}$, the histomorphometry $\mathrm{BV} / \mathrm{TV}_{2 \mathrm{D}}$, the trabecular $\mathrm{BMD}$ or the entire (trabecular bone plus cortical bone) BMD, can be used to quantify the amount of material within the bone.

It is important to compare the proposed measures of complexity with the measures obtained with the current "golden standard" for assessment of trabecular bone: traditional 2D histomorphometry.

The only 2D histomorphometric measure of trabecular bone structure that has a firm foundation in topology is connectivity density (CD), and it is therefore one of the most important histomorphometric measures. Among all analyzed measures including other parameters based on histomorphometry the best correlation to $\mathrm{CD}$ is provided by the 
complexity measures: SurfCI $\left(r_{s}=0.7\right)$ and Normalized Entropy of geometrical locations $\left(r_{s}=0.64\right)$.

Good correlation to the histomorphometric parameters quantifying the distribution of trabeculae, Tb.N, Tb.Sp, and to CD $\left(r_{s}=0.61,-0.76\right.$ and 0.7$)$ confirms that the Normalized Entropy is capable of assessing the bone geometrical composition in 3D. Another 3D structural parameter, SurfIGE correlates well with Tb.Th, Nd.Tm, and TBPf with correlation coefficient $r_{s}=-0.75,-0.72$, and 0.73 respectively.

The complexity measures SCI3D correlates to six, and SurfCI correlates to four histomorphometric measures simultaneously. Each of the histomorphometric parameters describes a different aspect of the organization of the bone material. A high correlation coefficient with a particular histomorphometric parameter indicates that the corresponding information is included in the considered measure of complexity.

SCI3D correlates: to the amount of bone material expressed by $3 \mathrm{D} \mathrm{BV} / \mathrm{TV}\left(r_{s}=0.94\right)$, to the geometry of trabecular bone network assessed by Tb.Th, Tb.N, Tb.Sp, Nd/Tm, $V_{\text {m.space }}^{*}\left(r_{s}=0.7,0.64,-0.8,0.76\right)$, to the distribution of material in 3D space quantified by the Normalized Entropy $S_{\text {norm }}$ and TBPf $\left(r_{s}=0.9,-0.79\right)$, as well as to the other complexity measures SurfCI and SurfIGE.

SurfCI includes information about the amount of bone material (quantified by BV/TV, $r_{s}=0.8$ ), the geometry of the construction (for Tb.N, Tb.Sp, and $V_{\text {m.space }}^{*} r_{s}=0.66$, $-0.76,-0.61$ ), and the connectivity of the trabeculae (expressed by CD, $r_{s}=0.7$ ). SurfCI values are also in excellent agreement $\left(r_{s}>0.91\right)$ with other 3D measures of complexity: SCI3D and SCI(BV/TV).

Measures of complexity are known to have the ability to quantify, accumulate, and combine different kinds of information $[14,32]$. The correlation we found between the complexity measures and the histomorphometric measures confirms that the new proposed 3D measures of complexity combine and assess different kinds of information and quantify the bone architecture in a holistic way.

The described technique of 3D bone assessment can be used for bone status evaluation from bone biopsies. This might be, for instance, of great interest for pharmacological studies of bone alterating drugs. It is not proposed to require bone biopsies for the assessment of the bone status of space-flying personnel; however, this study suggests to scrutinize the region of the proximal tibia further in order to establish it as a new site for radiological non-invasive bone evaluation.

\section{Conclusion}

We have further developed the technique of symbolic encoding and have proposed new measures of complexity to assess quantitatively the structural composition of bone tissue in 3D. Changes in trabecular bone composition during development of osteoporosis were used as a model for bone loss in microgravity conditions during a space flight. 3D struc- 
tural composition and deterioration of human bone tissue was assessed by analyzing 3D data sets of human tibial bone biopsies acquired by micro-CT. Despite that the study of the 3D measures of complexity is a part of an ongoing research project and is still work in progress, the results presented in this article provide new insights into the changes in bone structural composition:

1. The complexity of the bone architecture decreases while bone mass is lost.

2. Beyond a certain amount of bone loss, the relative probabilities of surface and internal bone voxels decrease at different rates.

3. The proposed measures provide a nonlinear holistic approach to quantify the amount, geometry, distribution, connectivity, and structural complexity of bone tissue. This conclusion is confirmed by a high correlation between these measures and the current "golden standard" for assessment of trabecular bone: traditional 2D histomorphometry.

Our findings suggest that the structural changes in bone tissue can be quantified with the proposed technique and may help to diagnose and monitor changes in bone structure of patients on Earth as well as of the space-flying personnel.

\section{Acknowledgment}

This study was made possible in part by grants from the Microgravity Application Program/Biotechnology from the Manned Spaceflight Program of the European Space Agency (ESA). The authors would also like to acknowledge Scanco Medical, Roche Pharmaceuticals, and Siemens AG for support of the study.

We thank the Anatomical Institute of the Humboldt University and the Free University Berlin, Germany for the provision of the specimens. The authors are grateful for the excellent technical assistance of Erika May, Free University Berlin, for the image pQCT acquisition and of Inger Vang Magnussen, University of Aarhus, Denmark for embedding and sectioning of the bone samples.

\section{References}

[1] A. I. Grigoriev, et all, Clinical and physiological evaluation of bone changes among astronauts after long-term space flights, Aviakosm. Ekolog. Med. 32 (1998) 21-25.

[2] M. F. Holick, Microgravity-induced bone loss — will it limit human space exploration?, Lancet 355 (2000) 1569-1570.

[3] L. Vico, P. Collet, A. Guignandon, M.-H. Lafage-Proust, T.Thomas, M. Rehalia, C. Alexandre, Effects of long-term microgravity exposure of cancellous and cortical weightbearing bones of cosmonauts, Lancet 355 (2000) 1607-1611.

[4] N. Rodionova, V. Oganov, O. Polkovenko, Mechanisms of gravity-dependent changes in the bone tissue, Journal of Gravitational Physiology 9 (1) (2002) 169-170, and ref. there in.

[5] Complex systems, Nature 410 (6825) (2001) 241-284. 
[6] R. Lakes, Materials with structural hierarchy, Nature 361 (1993) 511-515.

[7] G. B. Olson, Computational design of hierarchically structured materials, Science 277 (1997) $1237-1242$.

[8] S. Weinberg, Dreams of a final theory, Pantheon Books, New York, 1992.

[9] I. Stewart, Life's other secret, Wiley \& Sons, New York, 1998.

[10] D. C. Mikulecky, The emergence of complexity: science coming of age or science growing old?, Computers and Chemistry 25 (2001) 341-348.

[11] B.-L. Hao, Symbolic dynamic and characterization of complexity, Physica D 51 (1991) $161-176$.

[12] E. Ott, Chaos in Dynamical Systems, Cambridge University Press, Cambridge, 1993, p. 108.

[13] R. Wackerbauer, A. Witt, H. Atmanspacher, J. Kurths, H. Scheingraber, A comparative classification of complexity measures, Chaos, Solitons \& Fractals 4 (1) (1994) 133-173.

[14] P. Saparin, W. Gowin, J. Kurths, D. Felsenberg, Quantification of bone structure using symbolic dynamics and measures of complexity, Phys. Rev. E 58 (5) (1998) 6449-6459.

[15] W. Gowin, P. Saparin, J. Kurths, D. Felsenberg, Bone architecture assessment with measures of complexity, Acta Astronautica 49 (6-10) (2001) 171-178.

[16] P. Saparin, W. Gowin, D. Felsenberg, Comparison of bone loss with the changes of bone architecture at six different skeletal sites using measures of complexity, Journal of Gravitational Physiology 9 (1) (2002) 177-178.

[17] D. Sterio, The unbiased estimator of number and sizes of arbitrary particles using the disector, Journal of Microscopy 134 (1984) 127-136.

[18] J. Thomsen, E. Ebbesen, Li. Mosekilde, A new method of comprehensive static histomorphometry applied on human lumbar vertebral cancellous bone, Bone 27 (2000) $129-138$.

[19] A. Vesterby, Star volume of marrow space and trabeculae in iliac crest: Sampling procedure and correlation to star volume of first lumbar vertebra, Bone 11 (1990) 149-155.

[20] A. Parfitt, C. Mathews, A. Villanueva, M. Kleerekoper, B. Frame, D. Rao, Bone grafting from the proximal tibia, The Journal of Clinical Investigation 72 (1983) 1396-1409.

[21] N. Garrahan, R. Mellish, J. Compston, A new method for the two-dimensional analysis of bone structure in human iliac crest biopsies, Journal of Microscopy 142 (1986) 341-349.

[22] M. Hahn, M. Vogel, M. Pompesius-Kempa, G. Delling, Trabecular bone pattern factor - a new parameter for simple quantification of bone microarchitecture, Bone 13 (1992) 327-330.

[23] H. Gundersen, R. Boyce, J. Nyengaard, A. Odgaard, The conneulor: Unbiased estimation of connectivity using physical disector under projection, Bone 14 (1993) 217-222.

[24] V. Alt, A. Nawab, D. Seligson, Bone grafting from the proximal tibia, The Journal of Trauma 47 (1999) 555-557.

[25] C. Huges, P. J. Revington, The proximal tibia donor site in cleft alveolar bone grafting: Experience of 75 consecutive cases, Journal of Cranio-Maxillofacial Surgery 30 (2002) 12-16. 
[26] J. R.M. O'Keeffe, B. Riemer, S. Butterfield, Harvesting of autogenous cancellous bone graft from the proximal tibial metaphysis, Journal of Orthopaedic Trauma 5 (1991) 469-474.

[27] A. Zaikin, P. Saparin, S. Prohaska, J. Kurths, W. Gowin, Bone modeling and structural measures of complexity, Journal of Gravitational Physiology 9 (1) (2002) 175-176.

[28] S. Prohaska, H.-C. Hege, Fast visualization of plane-like structures in voxel data, in: IEEE Visualization 2002, IEEE, Boston, MA, USA, 2002, pp. 29-36.

[29] S. Prohaska, H.-C. Hege, M. Giehl, W. Gowin, Visual Analysis of Trabecular Bone Structure, Journal of Gravitational Physiology 9 (1) (2002) 171-172.

[30] C. E. Shannon, W. Weaver, The mathematical theory of communication, Univ. of Illinois Press, Urbana, 1949.

[31] J. Balatoni, A. Renyi, Selected Papers of A. Renyi, Vol. 1, Acadeiau, Budapest, 1976, p. 558.

[32] W. Gowin, P. Saparin, J. Kurths, D. Felsenberg, Measures of complexity for cancellous bone, Technology and Health Care 6 (1998) 373-389. 


\section{Figure Legends}

Fig. 1: Selection of the VOI inside the analyzed biopsy. Left: axial cross-section of a human tibia biopsy reveals the presence of residual drilling bone dust at the outer contour of the biopsy cylinder. Right: Dust-free VOI selected inside the biopsy (shown as opaque volume) excludes the peripheral regions containing the drilling residue.

Fig. 2: Calculation of 3D Normalized Entropy of geometrical locations. Left: The partition of the biopsy into 3D bins is used to calculate the value of the entropy of bone geometrical locations. Right: The partition of the VOI is used to estimate the maximal value of the entropy achievable for the given shape and size of the VOI and the given partition of the $3 \mathrm{D}$ space.

Fig. 3: Calculation of complexity indices, moving cubic window.

Fig. 4: A long biopsy was numerically segmented into $5 \mathrm{~mm}$ fragments which are shown in different colors. The cortical bone is at the left of the image, the internal area of the tibia is at the right.

Fig. 5: Changes of BV/TV (left) and SCI3D (right) along the biopsies. The horizontal axis represent the number of each $5 \mathrm{~mm}$ long segment. The results of the analysis of the three longest biopsies are shown.

Fig. 6: Decay of the Normalized Entropy (left) and the SCI3D (right) during bone loss plotted versus BV/TV for biopsies with different grades of osteoporosis. Circles represent the biopsies evaluation results, steady curves are the polynomial approximations.

Fig. 7: The Structure Complexity Index based on the distribution of local BV/TV ratios, $\mathrm{SCI}\left(\mathrm{BV} / \mathrm{TV}_{\text {loc }}\right)$, decreases $25 \%$ during the development of osteoporosis. Circles represent the biopsy evaluation results, solid curves are the polynomial approximations.

Fig. 8: Surface Complexity Index SurfCI (left) and Surface Index of a Global Ensemble SurfIGE (right) versus bone volume to total volume ratio BV/TV. Circles represent the biopsy evaluation results, solid curves are the polynomial approximations.

Fig. 9: Probabilities of bone surface voxels (filled triangles) [the polynomial approximation is shown by the dashed curve.] and internal bone voxels (filled circles) [the polynomial approximation is shown by the steady curve.] normalized by the total amount of bone voxels and plotted versus BV/TV. 

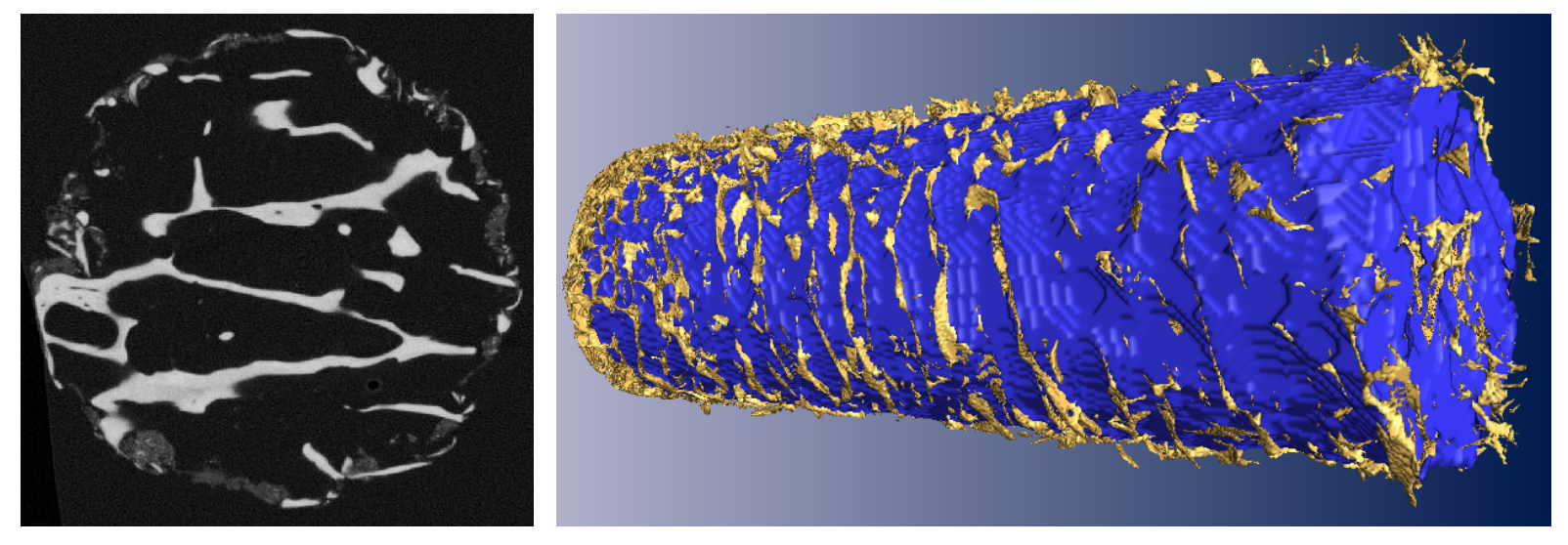

Fig. 1.
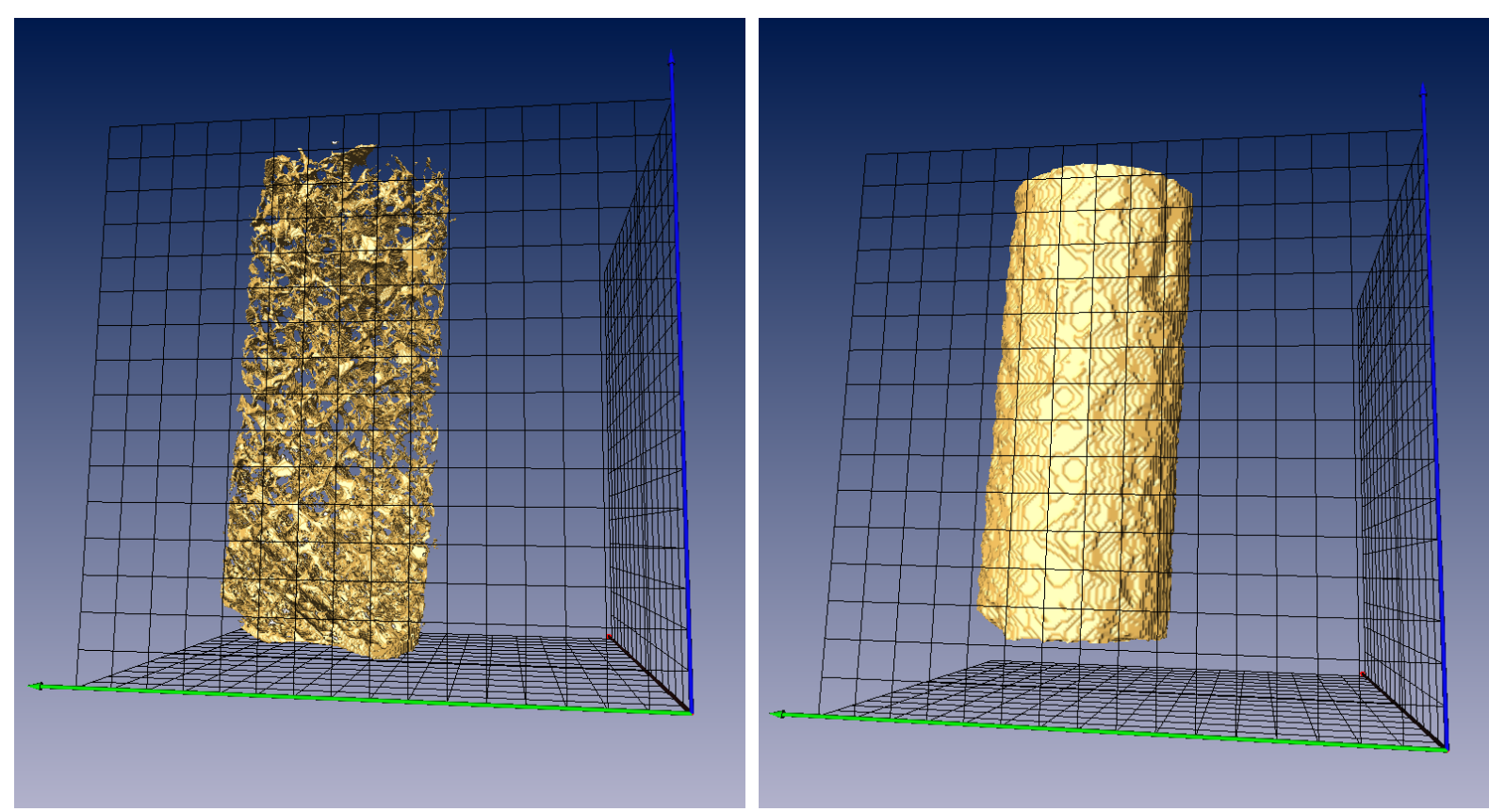

Fig. 2. 


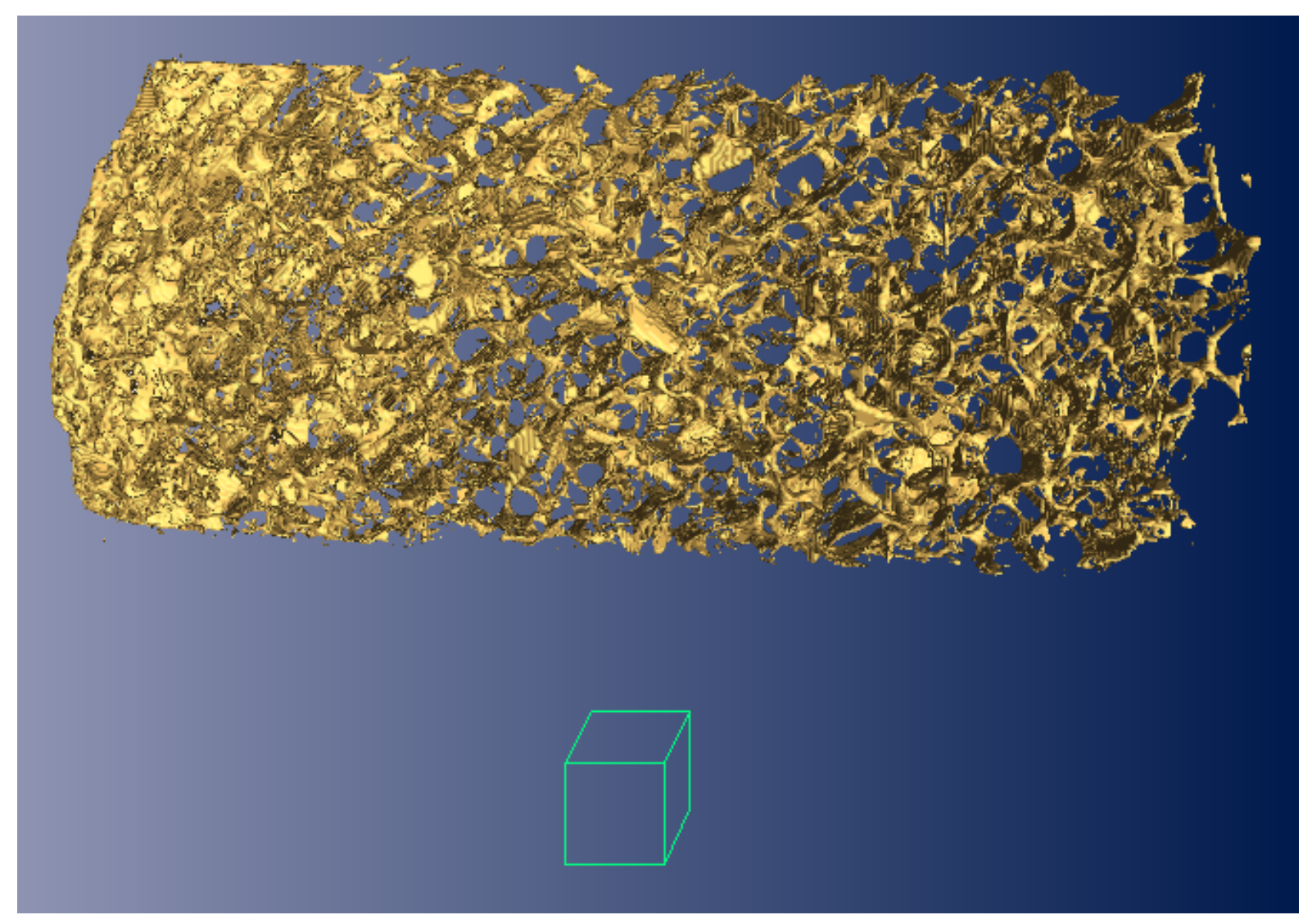

Fig. 3. 


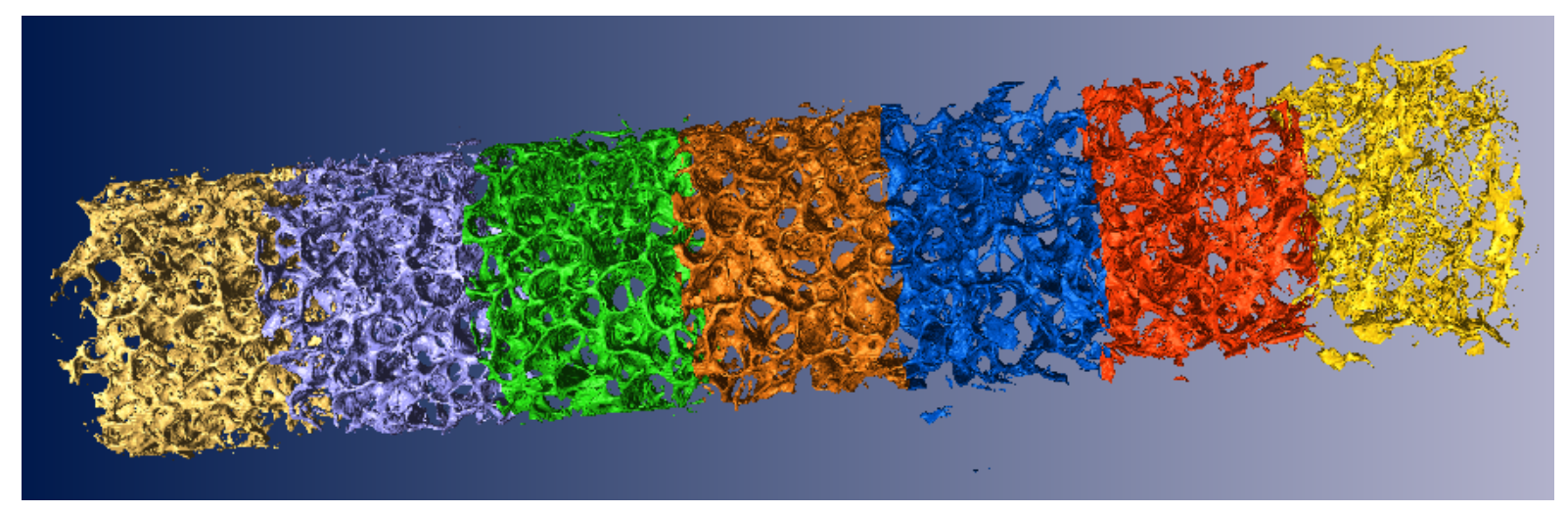

Fig. 4.
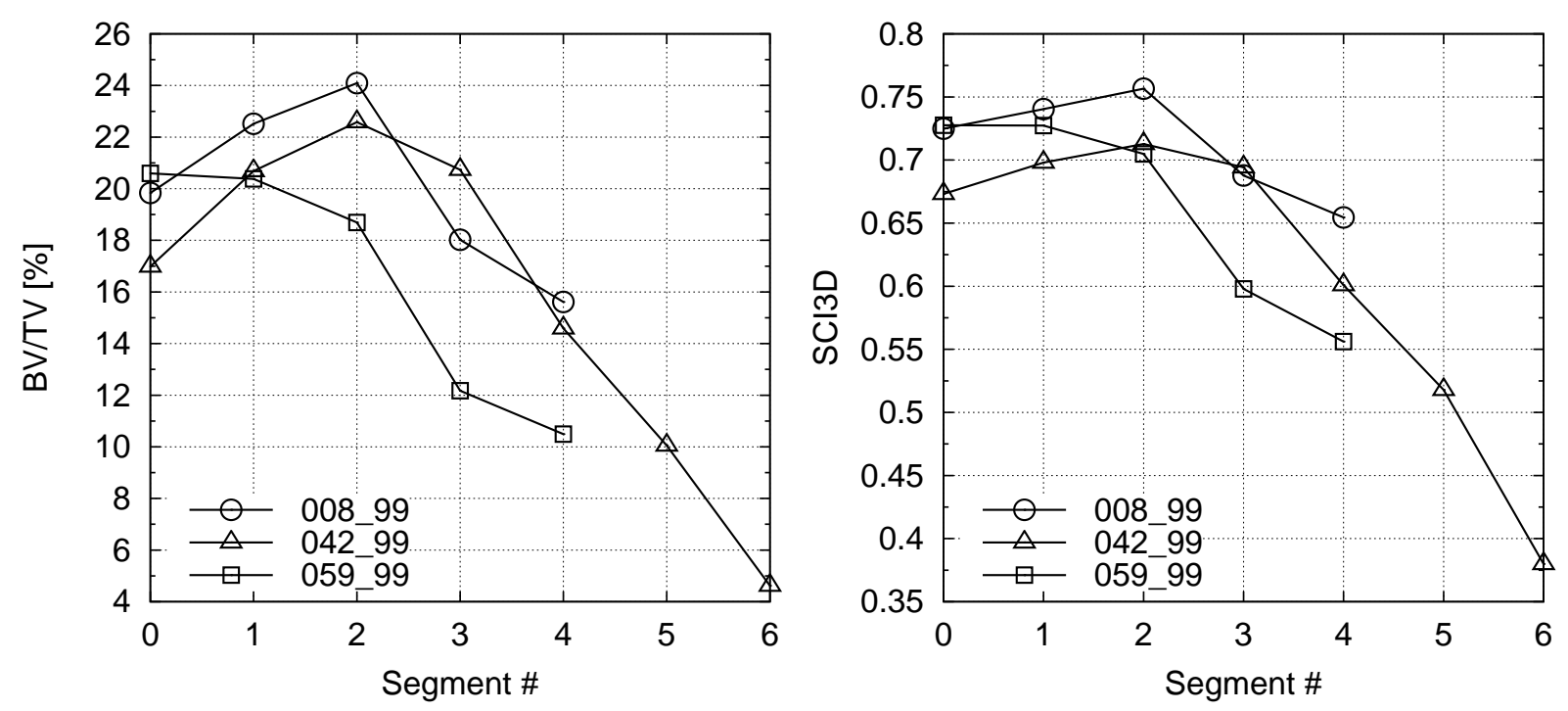

Fig. 5. 

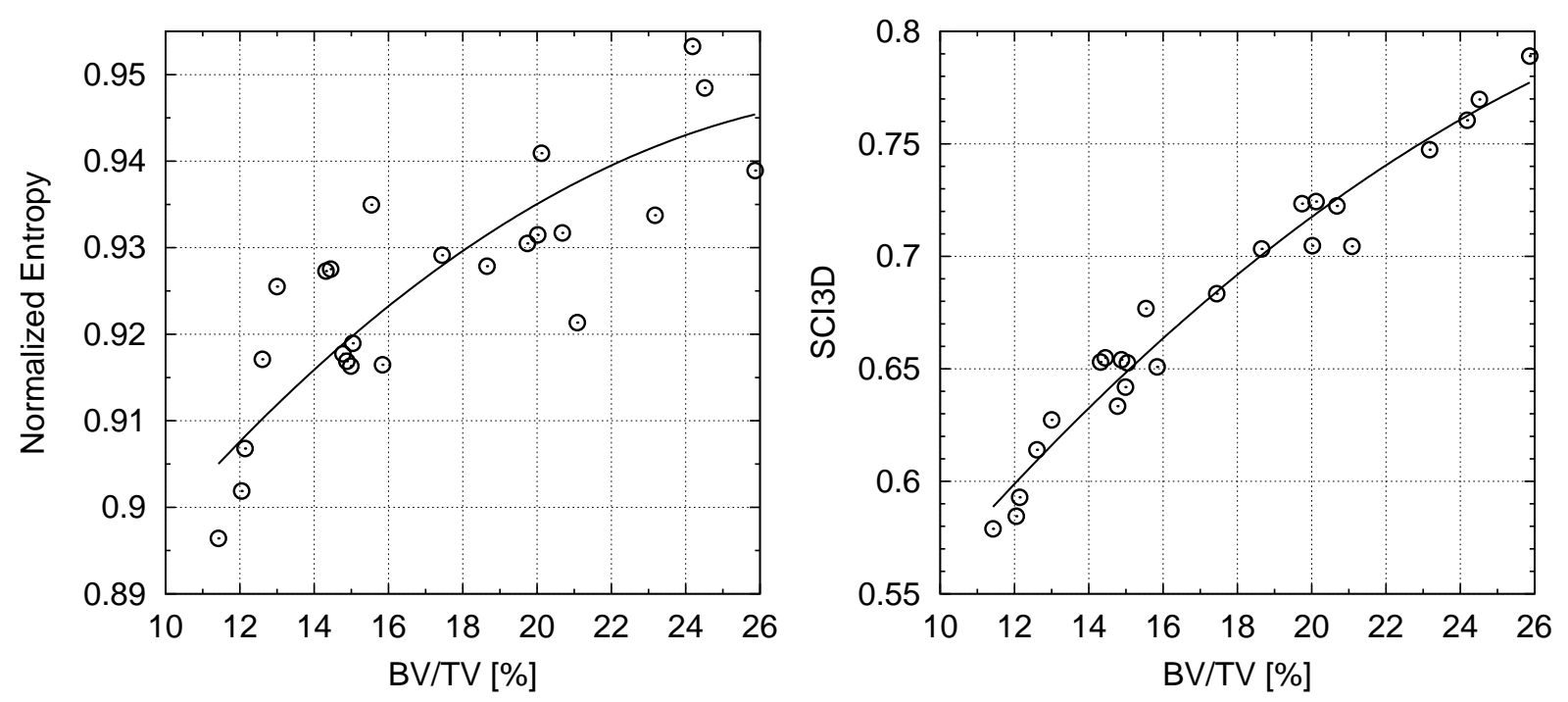

Fig. 6 .

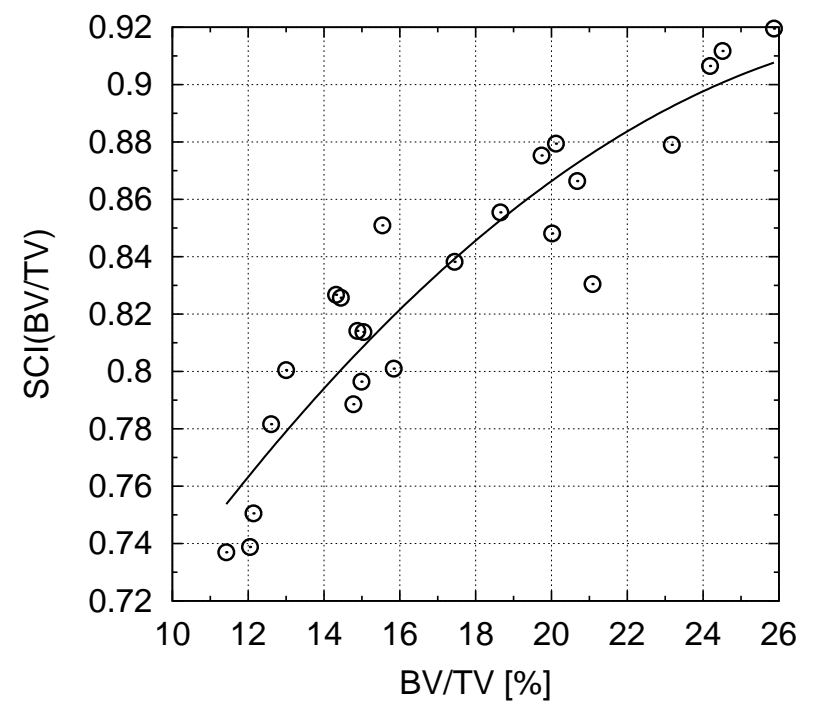

Fig. 7. 

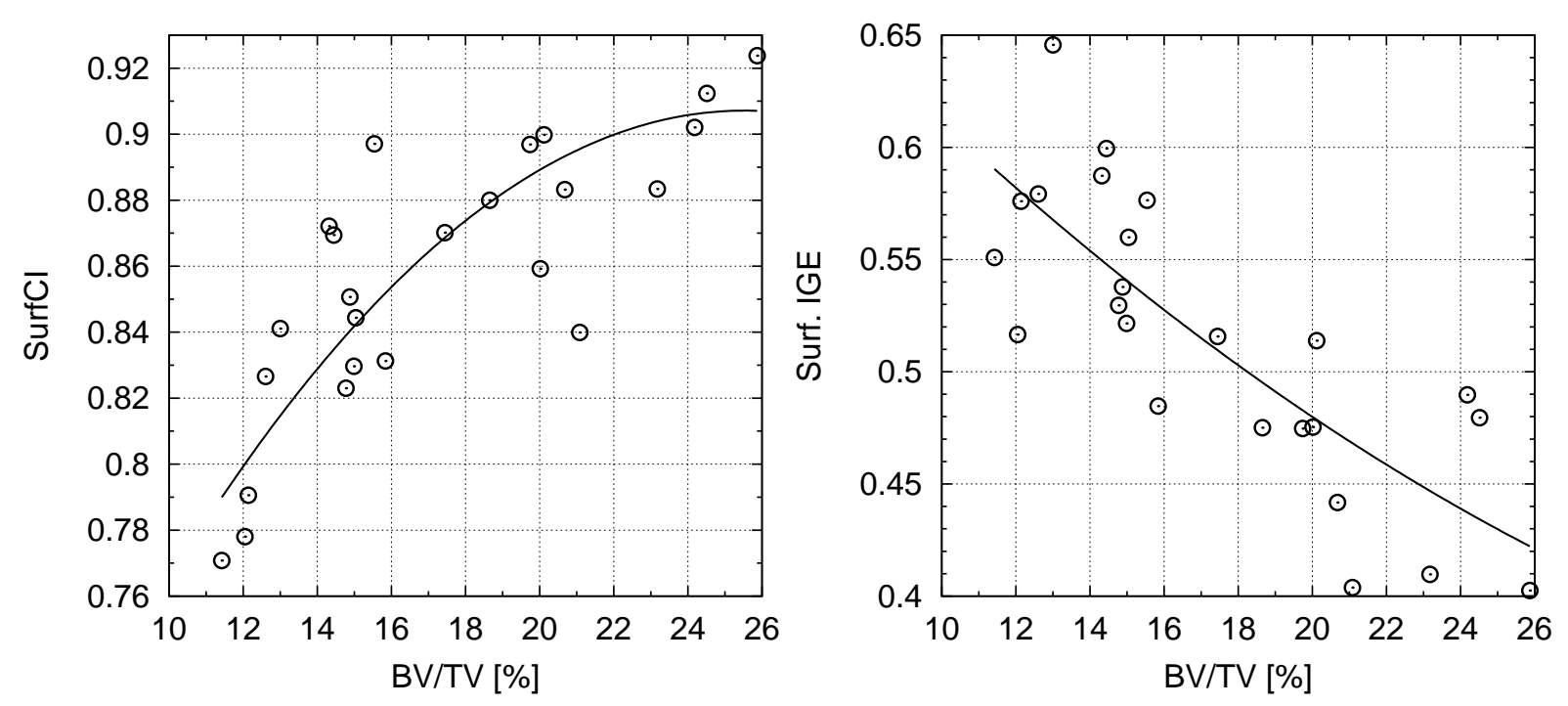

Fig. 8.

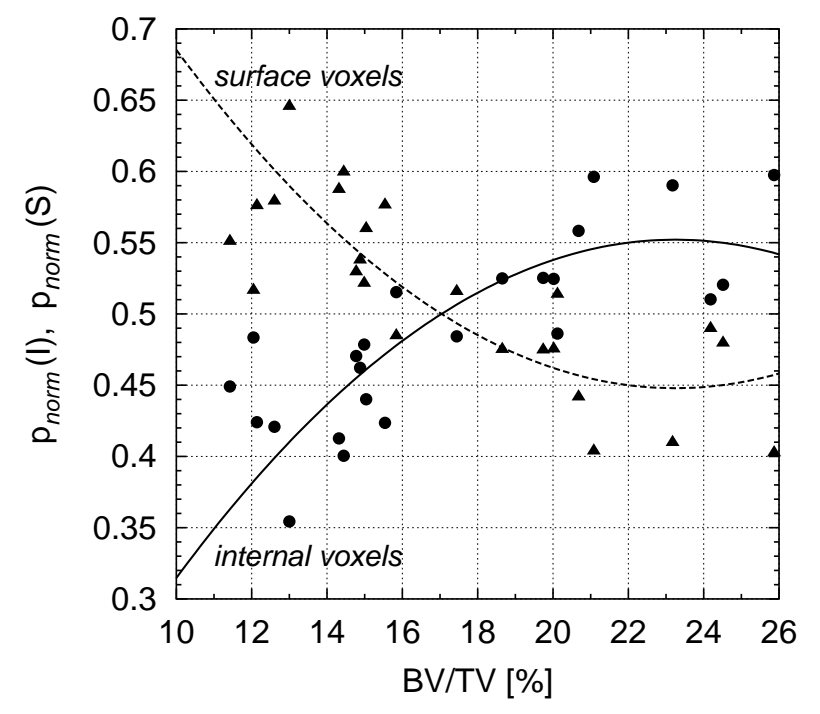

Fig. 9. 